\title{
A unified synthetic approach to the pyrazinone dragmacidins
}

\author{
Neil K. Garg and Brian M. Stoltz* \\ Received (in Cambridge, UK) 26th April 2006, Accepted 15th June 2006 \\ First published as an Advance Article on the web 18th July 2006 \\ DOI: $10.1039 / \mathrm{b} 605929 \mathrm{e}$
}

This review describes recent developments from our laboratory involving the synthesis of the structurally complex, pyrazinone-containing dragmacidin alkaloids.

\section{Introduction}

Over the past several decades, the search for natural products in marine and terrestrial environments has led to the discovery of a number of biologically active bis(indole) alkaloids. ${ }^{1}$ These compounds, as well as their unnatural analogs, have shown promise as leads for the development of novel therapeutics. Although many bis(indole) alkaloids have been found in nature, relatively few have been discovered in marine environments. ${ }^{2}$ Of those, a family of compounds isolated from Dragmacidon sp. (i.e., the dragmacidins) have received considerable attention from the scientific community over the past decade because of their broad range of biological activity and complex and unique structures (1-7, Fig. 1). ${ }^{3,4}$ The four dragmacidins initially identified (1-4) contain a piperazine linker and display modest antifungal, antiviral and cytotoxic activities. ${ }^{3 a-c}$ However, our interest in these natural products was piqued by the more complex pyrazinone-containing family members, dragmacidins D (5), E (6), and F (7). ${ }^{3 d-g}$ The striking array of functionality in these stunning molecules (i.e., the pyrazinone, aminoimidazole, aryl bromide, the free phenol in

Division of Chemistry and Chemical Engineering, California Institute of Technology, Pasadena, CA, 91125,USA. E-mail: stoltz@caltech.edu; Fax: 626-564-9297; Tel: 626-395-6064

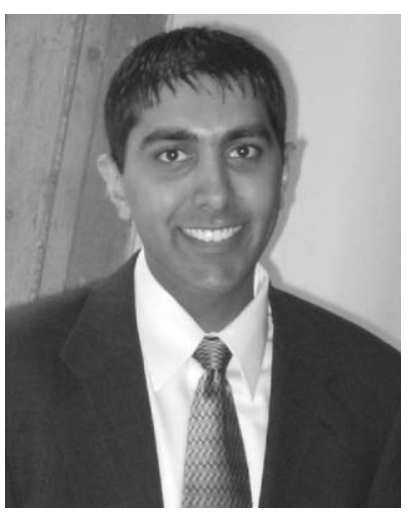

Neil K. Garg
Neil K. Garg was born in Fishkill, NY, USA in 1978. He received his $B S$ in Chemistry from New York University where he did undergraduate research with Professor Marc Walters. During his undergraduate years, he spent several months in Strasbourg, France while conducting research with Professor Mir Wais Hosseini at Université Louis Pasteur. Dr Garg received his $P h D$ in March, 2005 in Professor Brian Stoltz's group at the California Institute of Technology, where his studies were focused on the total synthesis of the dragmacidin alkaloids. $\mathrm{Dr}$ Garg is currently a postdoctoral scholar in the laboratory of Professor Larry E. Overman at the University of California, Irvine.
5), in addition to the architectural arrangement of the polyheterocyclic core structures, posed a variety of synthetic challenges.

Preliminary studies suggest that the pyrazinone dragmacidins (i.e., 5-7) are important compounds from a biological standpoint, thereby rendering them attractive targets for total synthesis. In particular, dragmacidins D (5) and F (7) have been studied most extensively. In addition to displaying antiinflammatory activity, cytotoxicity and phosphatase inhibition, ${ }^{3 d, 3 e} \mathbf{5}$ has shown selectivity as an inhibitor of neural nitric oxide synthase. ${ }^{5}$ Molecules possessing this bioactivity are thought to be excellent leads for the discovery of novel drugs that target neurodegenerative disorders such as Alzheimer's, Parkinson's and Huntington's diseases. ${ }^{6}$ Furthermore, dragmacidin F (7) is reported to exhibit in vitro antiviral activity against herpes simplex virus (HSV-I; $\left.\mathrm{EC}_{50}=95.8 \mu \mathrm{M}\right)$ and human immunodeficiency virus (HIV-I; $\left.\mathrm{EC}_{50}=0.91 \mu \mathrm{M}\right){ }^{3 f}$ Given the difficulty in obtaining dragmacidins from marine environments, as well as the low abundance of some of these compounds from natural sources, we reasoned that synthetic routes to the pyrazinone dragmacidins (5-7) and their analogs could facilitate advanced biological studies.

In this review, we highlight recent synthetic studies from our laboratory that has led to the development of a unified strategy

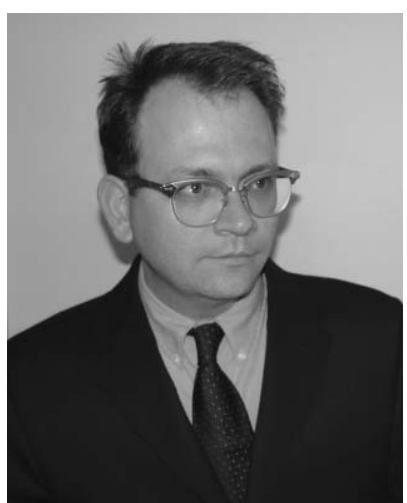

Brian M. Stoltz
Brian M. Stoltz was born in Philadelphia, Pennsylvania, USA in 1970. After spending a year at the Ludwig Maximilians Universität in München, Germany, he obtained his BS in Chemistry and $B A$ in German from Indiana University of Pennsylvania in 1993. He then earned his PhD in 1997 under the direction of Professor John L. Wood at Yale University, and following an NIH postdoctoral fellowship in the laboratories of Professor E.

J. Corey at Harvard University (1998-2000), he joined the faculty at the California Institute of Technology in 2000. His research focuses on the design and implementation of new synthetic strategies for the synthesis of complex molecules possessing important biological properties, in addition to the development of new synthetic methods including asymmetric catalysis and cascade processes. 

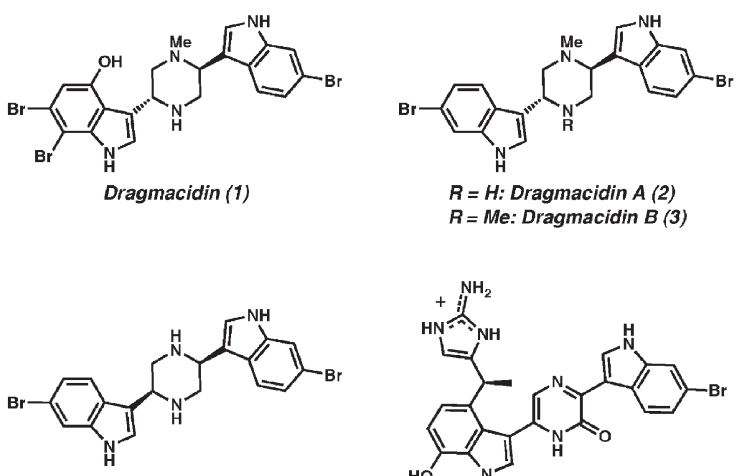

Dragmacidin C (4)
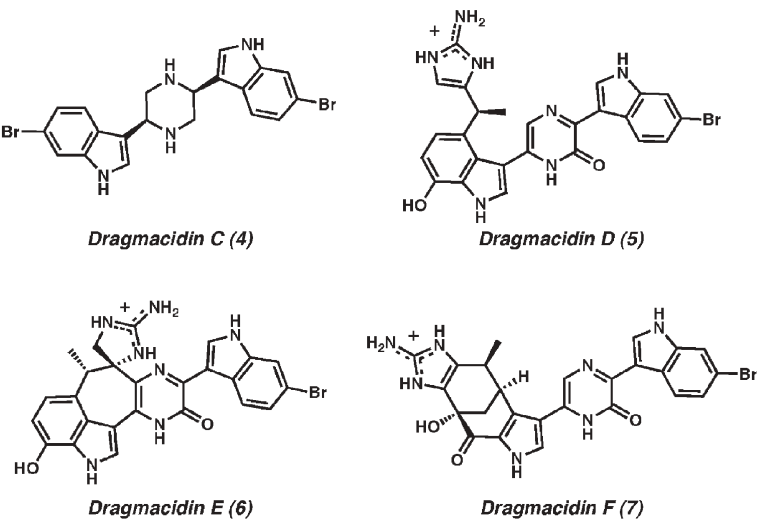

Fig. 1 The dragmacidin alkaloids.

for the preparation of several dragmacidin alkaloids. The success of these efforts can largely be attributed to the power of transition metal chemistry in modern organic synthesis.

\section{The design of a unified strategy}

While the biosynthesis of the dragmacidins has not been studied in detail, dragmacidins D, E and F are likely biosynthetically related. Of the possible biosynthetic scenarios, most probable is that dragmacidins E (6) and F (7) are derived by cyclization of either dragmacidin D (5) or a closely related congener (Fig. 2). For example, dragmacidins D and E are structural isomers that differ by a single $\mathrm{C}-\mathrm{C}$ bond. In nature, it is likely that a Friedel-Crafts cyclization between the pyrazinone and aminoimidazole groups of dragmacidin D occurs in order to construct the seven-membered ring of dragmacidin E (i.e., 5 $\rightarrow \mathbf{6}$ ). Dragmacidins D (5) and F (7) also differ in connectivity by one $\mathrm{C}-\mathrm{C}$ bond; however, in this case, there is also a difference in oxidation state between the two

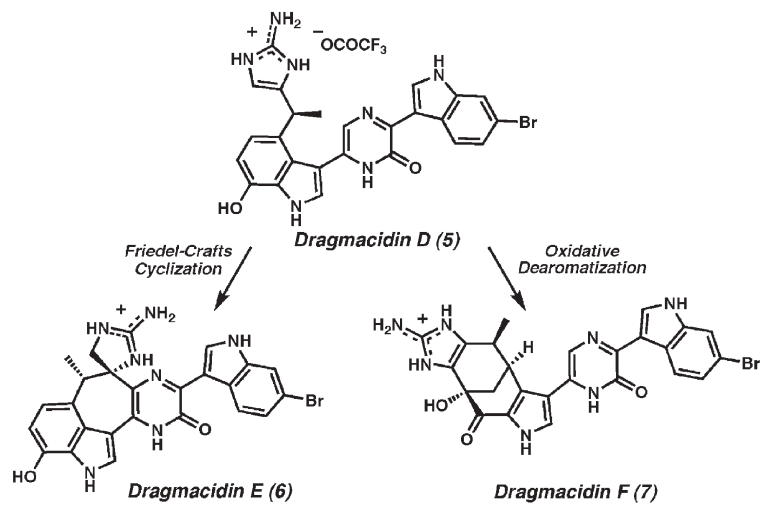

Fig. 2 Unified biosynthetic relationship. natural products. Thus, oxidative dearomatization with concomitant cyclization could facilitate the formation of the unique polycyclic framework present in dragmacidin $\mathrm{F}$ (i.e., $5 \rightarrow 7)$. Related oxidation pathways for tryptophan derivatives have been observed in nature. ${ }^{7}$ Finally, it is plausible that dragmacidin D and hence the core of all the dragmacidins are derived by the biosynthetic dimerization of two functionalized tryptophan derivatives.

The identification that there was a clear biosynthetic relationship between the dragmacidins led us to consider the design of a unified synthetic strategy that would take advantage of the similar functionality groupings and connectivities of key skeletal components. While there were clear differences among the members of the pyrazinone family, the general feature that the molecules were constructed of a core pyrazinone flanked by two different heterocycles was of paramount importance in developing our strategy.

\section{The total synthesis of dragmacidin D}

Having selected the pyrazinone-containing dragmacidins, namely, dragmacidins D (5), E (6) and F (7), as formidable synthetic targets for our laboratory we first chose to pursue the total synthesis of dragmacidin D (5), ${ }^{3 d, e, 8}$ predominantly because it appeared to be the simplest of the pyrazinonecontaining family members. Learning how to selectively build the skeleton of dragmacidin D, as well as construct and reveal all of the functionality present, was a major challenge to our laboratory. Moreover, we hoped to develop a strategy for the preparation of dragmacidin D (5) that would be amenable to the synthesis of the other complex dragmacidin natural products.

When considering the structure of dragmacidin D (5), several synthetic challenges become apparent. Dragmacidin D possesses a total of seven nitrogen atoms, three of which are incorporated in the aminoimidazole moiety, while two are within the pyrazinone core. The compound contains an unusual bis(indole) architecture featuring a 3,4,7-trisubstituted indole and a 3,6-disubstituted indole. Access to both of these indole substitution patterns is known to be synthetically challenging. ${ }^{9}$ It was predicted that dragmacidin D (5), as well as many of its synthetic precursors, would be highly polar, extremely reactive and perhaps difficult to handle in a laboratory setting.

Two retrosynthetic strategies for the synthesis of dragmacidin D (5) are presented in Scheme 1. We targeted 5 through

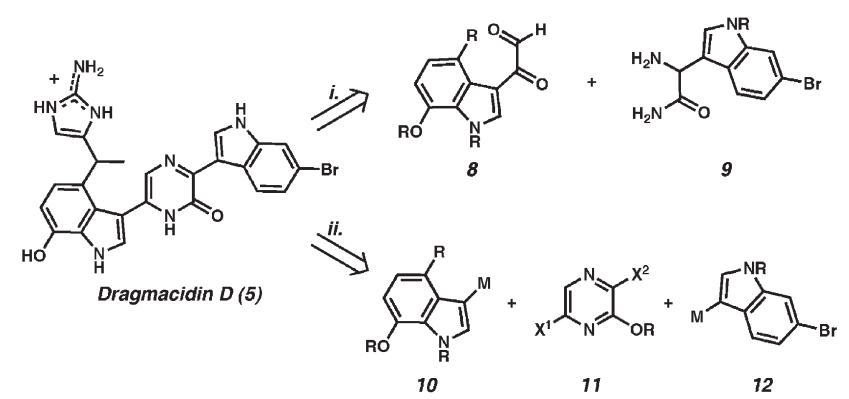

Scheme 1 Retrosynthesis of dragmacidin D (5). 
two complementary routes: (i) a classical cyclocondensation approach $^{10}$ and (ii) a more modern transition metal-mediated cross-coupling approach. ${ }^{11}$ In approach $i$, the pyrazinone system would be constructed through the linkage of two functionalized indole units $(\mathbf{8}+\mathbf{9})$, while in route $i i$, the dragmacidin core was envisioned to arise by a stepwise threecomponent coupling sequence $(\mathbf{1 0}+\mathbf{1 1}+\mathbf{1 2})$.

In 2000, our laboratory began working on the two approaches outlined above. Initially we investigated the viability of the cyclocondensation strategy (i). Although we were able to prepare simple bis(indole) pyrazinone model systems, attempts to utilize more advanced fragments were unsuccessful and we quickly redirected our attention to the transition metal-catalyzed cross-coupling route (ii). To carry out this plan successfully, it seemed that a more detailed strategy was needed, involving not only the exact order of the coupling reactions, but also the specific identity of each substrate involved. The judicious selection of halides, metals and protecting groups would be crucial. We thus carried out a series of experiments that would delineate suitable conditions for cross-coupling.

After examining a number of model systems, we began to identify appropriate substitution patterns for building blocks 10, 11 and 12 (Fig. 3). It was immediately clear that the use of $\operatorname{Pd}(0)$-catalyzed Suzuki couplings would be favorable given the abundant precedence for these cross-couplings in heterocycle chemistry. ${ }^{12}$ Thus, boron substituents were employed as the nucleophilic component for both indole substrates $(\mathbf{1 0} \approx \mathbf{1 3}$ and $12 \approx 15$ ). In addition, the protecting groups for the indoles were chosen in a manner that optimized orthogonality with respect to deprotection, thus facilitating control during late-stage manipulations. In particular, the $\mathrm{N}$-protecting groups were carefully selected. ${ }^{13}$ The SEM group of 13 was considered ideal due to its marked stability and electrondonating nature, ${ }^{14}$ while the Ts group of $\mathbf{1 5}$ was preferred mainly because of its success in model system studies. Perhaps the most important decision was the selection of halogens $\mathrm{X}^{1}$ and $\mathrm{X}^{2}$ of generic pyrazine 11. In order to keep 15 in tact through the cross-coupling sequence, both $\mathrm{X}^{1}$ and $\mathrm{X}^{2}$ would have to be more reactive to $\operatorname{Pd}(0)$ than the 6-bromoindole functionality. Ultimately, bromoiodopyrazine $\mathbf{1 4}$ was chosen as the key synthetic fragment that would allow for this selectivity. ${ }^{15}$ We then developed rapid syntheses of the three

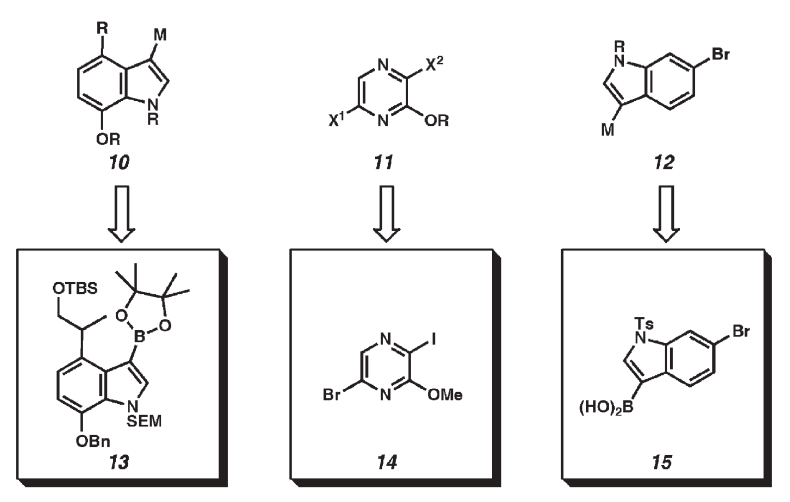

Fig. 3 Identification of cross-coupling fragments.

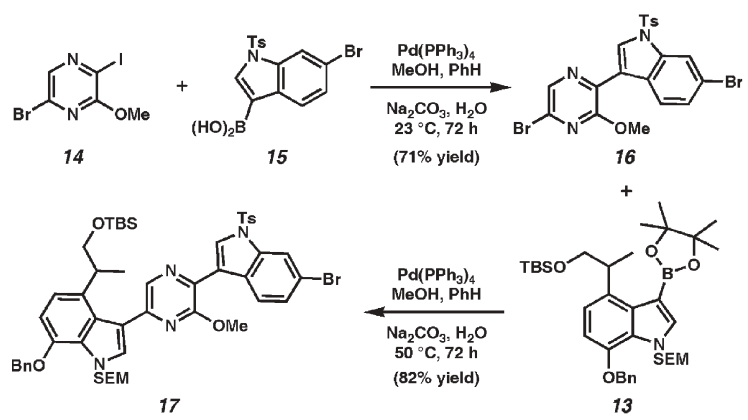

Scheme 2 Halogen-selective Suzuki couplings to access 17.

essential pieces $(\mathbf{1 3}, 14$ and $\mathbf{1 5})$ in a relatively straightforward manner.

With the appropriate fragments in hand (13, 14 and 15), the critical three-component coupling reaction sequence was explored. Suzuki coupling of dihalopyrazine 14 and indole 15 proceeded selectively to afford the coupled indolylpyrazine 16 (Scheme 2). In the second Suzuki coupling of dibromide 16 with boronic ester 13, we were delighted to find that the desired bis(indole)pyrazine $\mathbf{1 7}$ formed in good yield and with complete selectivity for coupling of the pyrazinyl bromide in the presence of the indolyl bromide. Precise temperature control was critical for the success of both coupling reactions (23 and $50{ }^{\circ} \mathrm{C}$, respectively). Importantly, the selectivity of the second Suzuki reaction depended not only on temperature, but also on the exact identity of each coupling substrate. In fact, varying protective groups on the indole nitrogen in $\mathbf{1 5}$ had a dramatic effect on halide reactivity, as competitive coupling of the indolyl bromide occurred when electron-donating $\mathrm{N}$-protective groups were employed.

In our initial endgame approach, we chose to first attempt installation of the aminoimidazole unit and proceeded by elaborating 17 to carboxylic acid 18 (Scheme 3). Subsequent conversion of $\mathbf{1 8}$ to bromoketone $\mathbf{1 9}$ was accomplished by an
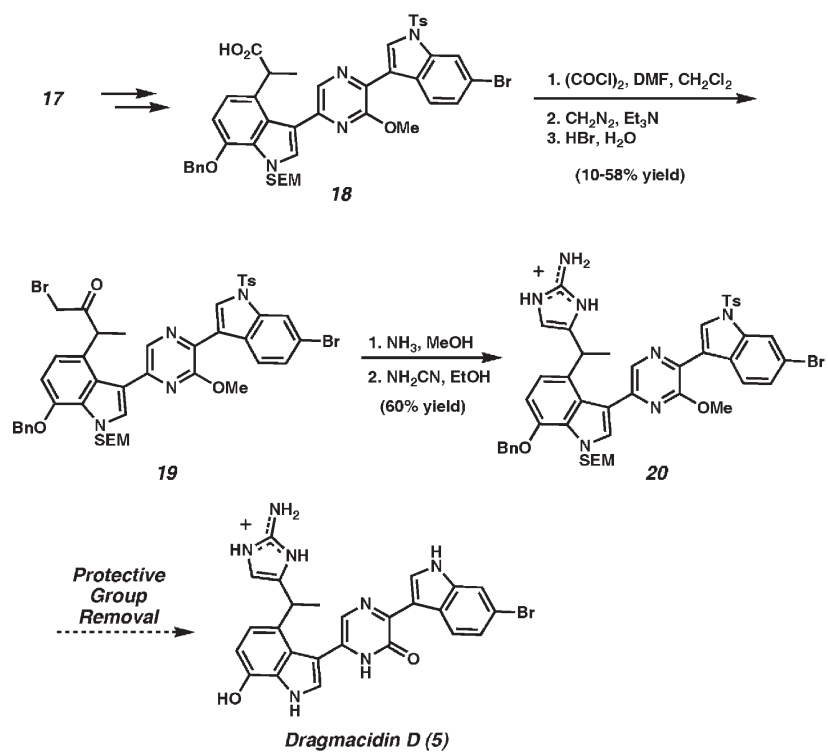

Scheme 3 End-game strategy 1. 
Arndt-Eistert-type homologation, followed by treatment with aqueous $\mathrm{HBr}$. The extreme sensitivity of the intermediate acid chloride was particularly troublesome and required that the diazomethane used in the reaction sequence be dried thoroughly over both potassium hydroxide and sodium metal immediately before use. ${ }^{16}$ In addition, chemical yields for this homologation varied to a large extent and caused substantial material throughput problems. Nonetheless, exposure of 19 to ammonia afforded an intermediate aminoketone which, in turn, underwent facile condensation with cyanamide to produce the desired aminoimidazole product (20). ${ }^{17}$ At this point, all that remained in order to complete the total synthesis of dragmacidin D (5) was the removal of the four protective groups from 20. Despite several months of experimentation, our efforts to complete the total synthesis of $\mathbf{5}$ were accompanied by decomposition of the aminoimidazole moiety, which was exceptionally unstable to the conditions (acidic, basic or reductive) needed to remove the protective groups that we had strategically chosen (vide supra).

The possibility of installing the aminoimidazole as the last step of the total synthesis, after the full deprotection of a latestage intermediate, was explored next. In addition, we sought an alternative one-carbon homologation reaction in place of the unreliable and experimentally tedious Arndt-Eistert sequence. After extensive experimentation, a solution to our problems was discovered. Namely, we found that nitromethane addition ${ }^{18}$ to aldehyde 21 and subsequent oxidation produced 22 in high yield (Scheme 4). Nitroketone 22, in turn, was a suitable intermediate for the removal of the indole nitrogen protective groups. Deoxygenated ethanolic potassium hydroxide facilitated removal of the $N$-tosyl group, ${ }^{19}$ while lithium tetrafluoroborate followed by aqueous sodium hydroxide effected complete hydrolysis of the SEM group $(\mathbf{2 2} \rightarrow \mathbf{2 3})$. Reduction of nitroketone $\mathbf{2 3}$ with stannous chloride, ${ }^{20}$ then cleavage of the benzyl and methyl ethers with iodotrimethylsilane revealed fully deprotected aminoketone $24 .^{21,22}$ Final installation of the aminoimidazolium unit occurred by treatment of $\mathbf{2 4}$ with cyanamide followed by trifluoroacetic acid
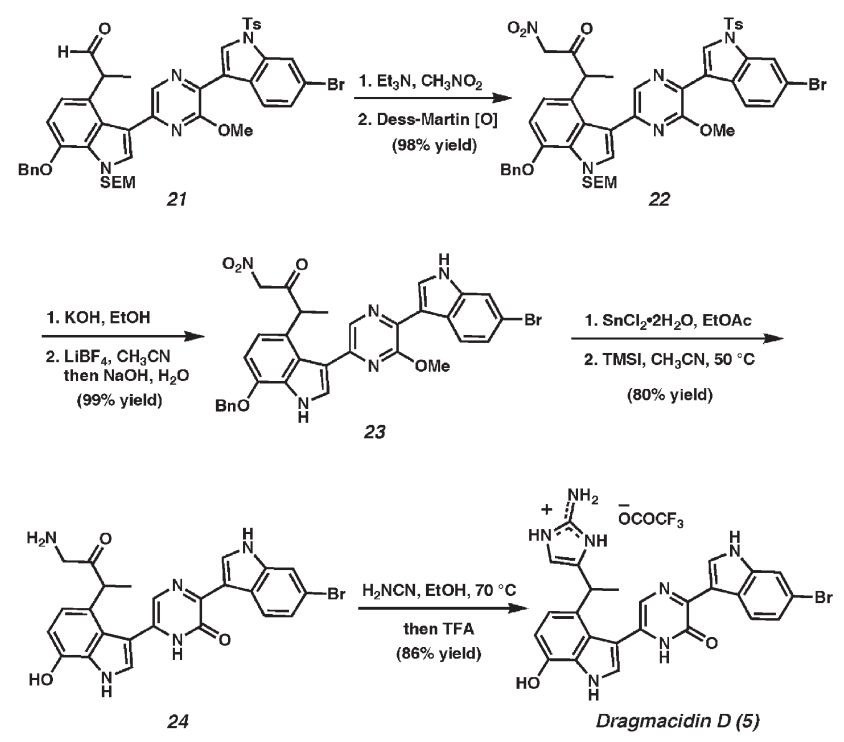

Scheme 4 The total synthesis of dragmacidin D (5). workup to produce dragmacidin D (5) in 86\% yield. This effort stands as the only completed total synthesis of dragmacidin D to date. ${ }^{23-25}$

\section{The total synthesis of dragmacidin $\mathbf{F}$}

Having developed a strategy to construct the bis(indole)pyrazinone core of dragmacidin D (5), we set out to apply our $\operatorname{Pd}(0)$ halogen-selective Suzuki coupling methodology to the synthesis of related natural products. We hypothesized that our approach could be amenable to the preparation of the antiviral agent dragmacidin $\mathrm{F},{ }^{3 f, g}$ which is perhaps the most challenging target of the dragmacidin natural products.

Dragmacidin F shares a number of structural features with dragmacidin D, namely, an aminoimidazolium fragment, a differentially substituted pyrazinone and a 6-bromoindole unit. However, rather than containing two indole functionalities, dragmacidin $\mathrm{F}$ possesses one indole, in addition to a pyrrole appended [3.3.1] bicycle. It is important to note that at the time of our synthetic effort, the absolute stereochemistry of natural dragmacidin F was not know; thus, the absolute stereochemistry of our target was chosen arbitrarily.

Our retrosynthetic analysis for dragmacidin $\mathrm{F}$ is shown in Scheme 5. We reasoned that the aminoimidazole would best be incorporated at a late stage in the synthesis. The carbon scaffold of 7, could be assembled via a series of halogenselective Suzuki cross coupling reactions of 25, 14 and 15 in an analogous manner to the dragmacidin D sequence. Since pyrazine $\mathbf{1 4}$ and boronic acid $\mathbf{1 5}$ were readily accessible, our success would depend highly on the ability to prepare pyrroloboronic ester 25. We envisioned deriving this key coupling fragment from parent bicycle $\mathbf{2 6}$ which, in turn, would arise via an intramolecular Pd-mediated oxidative carbocyclization reaction of acyl pyrrole 27. ${ }^{26}$ This latter transformation was extremely appealing to us given our laboratory's interest in $\mathrm{Pd}$ oxidation chemistry. ${ }^{27}$ Although we had previously cyclized indoles and electron rich aromatics in the presence of $\mathrm{Pd}(\mathrm{II})$, examples of pyrroles undergoing the
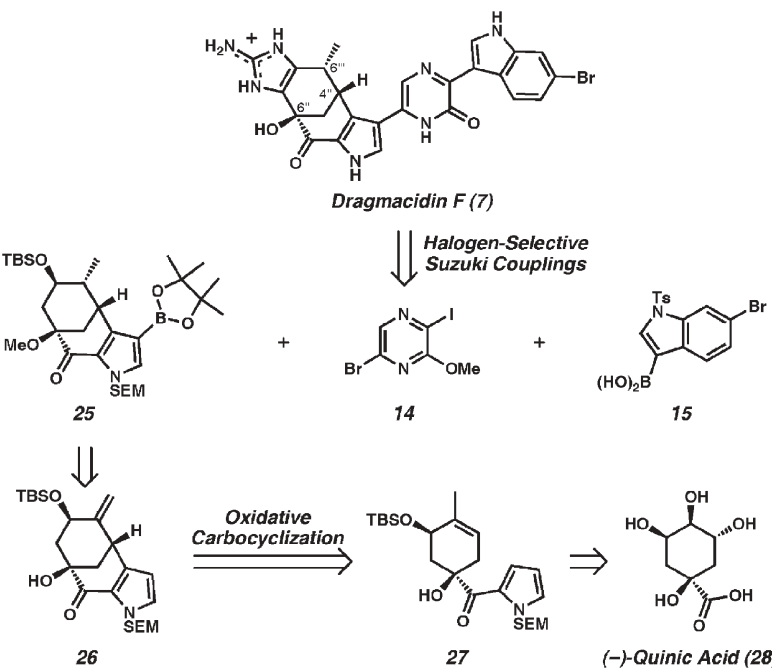

Scheme 5 Retrosynthesis of dragmacidin F (7). 

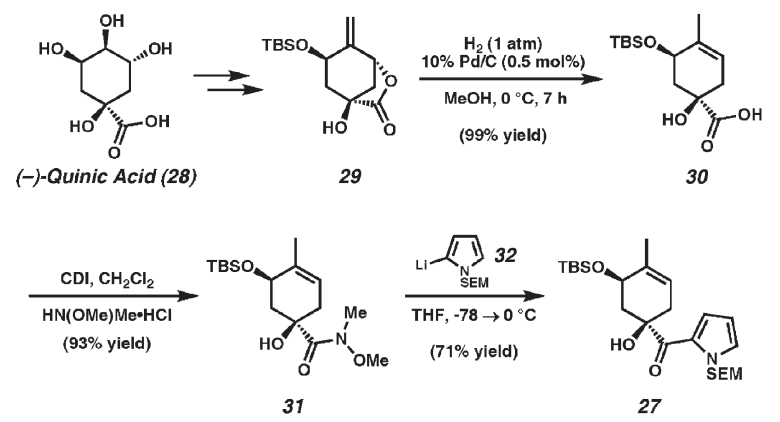

Scheme 6 Synthesis of cyclization substrate 27.

analogous carbocyclization were not known in the literature or our own laboratory. ${ }^{28}$ In the final retrosynthetic disconnection, pyrrole 27 would be accessible from (-)-quinic acid (28), ${ }^{29}$ a commercially available enantiopure starting material.

Our first task was to devise an efficient route to $\mathbf{2 7}$ beginning from (-)-quinic acid (28, Scheme 6). Lactone 29 could be obtained in four simple steps and then reductively isomerized to acid 30 upon treatment with $\mathrm{Pd} / \mathrm{C}$ in $\mathrm{MeOH}$ at $0{ }^{\circ} \mathrm{C}$ in the presence of $\mathrm{H}_{2}{ }^{30}$ Notably, this transformation proceeded in essentially quantitative yield and allowed for the preparation of multigram quantities of $\mathbf{3 0}$ in a facile manner. More importantly, acid $\mathbf{3 0}$ could be converted to cyclization substrate 27 via a two-step protocol involving Weinreb amide formation $^{30,31}$ and displacement by 2-lithio-1-SEM-pyrrole (32). ${ }^{31}$

With acyl pyrrole $\mathbf{2 7}$ in hand, we turned our attention to the key Pd(II)-mediated cyclization reaction (Scheme 7). After substantial experimentation, we found that [3.3.1] bicycle $\mathbf{2 6}$ could be formed upon exposure of 27 to a $\mathrm{Pd}(\mathrm{OAc})_{2} / \mathrm{DMSO}$ system. ${ }^{32}$ Under our optimized reaction protocol, bicycle $\mathbf{2 6}$ was isolated in $74 \%$ yield. Notably, this transformation $(\mathbf{2 7} \rightarrow \mathbf{2 6})$ results in functionalization of the electronically deactivated and sterically congested $\mathrm{C}(3)$ position of acyl pyrrole 27. ${ }^{33,34}$ Furthermore, undesired [3.2.2] bicycle 33 was not observed under these Pd(II) conditions, although 33 formed in substantial quantities in the analogous Heck reaction. ${ }^{35,36}$

Our next goal was to construct the carbon skeleton of the natural product (Scheme 8). Following a straightforward four step sequence, bicycle $\mathbf{2 6}$ was elaborated to the crucial boronic ester fragment (25). By analogy to our dragmacidin D studies, we were pleased to find that upon treatment of $\mathbf{2 5}$ with bromopyrazine 16 under our standard Suzuki coupling conditions, the desired $\mathrm{C}-\mathrm{C}$ bond formation took place to afford the fully coupled product (34) in $77 \%$ yield. Importantly, the indolyl bromide moiety was maintained under these reaction conditions. Following cleavage of the silyl ether and subsequent oxidation, we arrived at ketone $\mathbf{3 5}$.

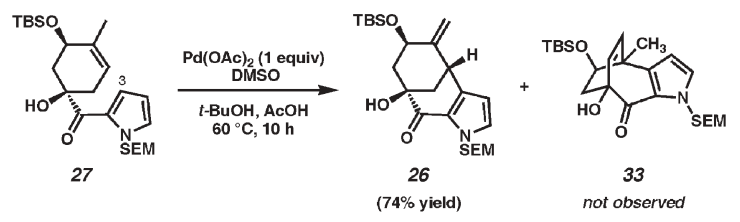

Scheme 7 Pd(II)-Mediated oxidative carbocyclization.
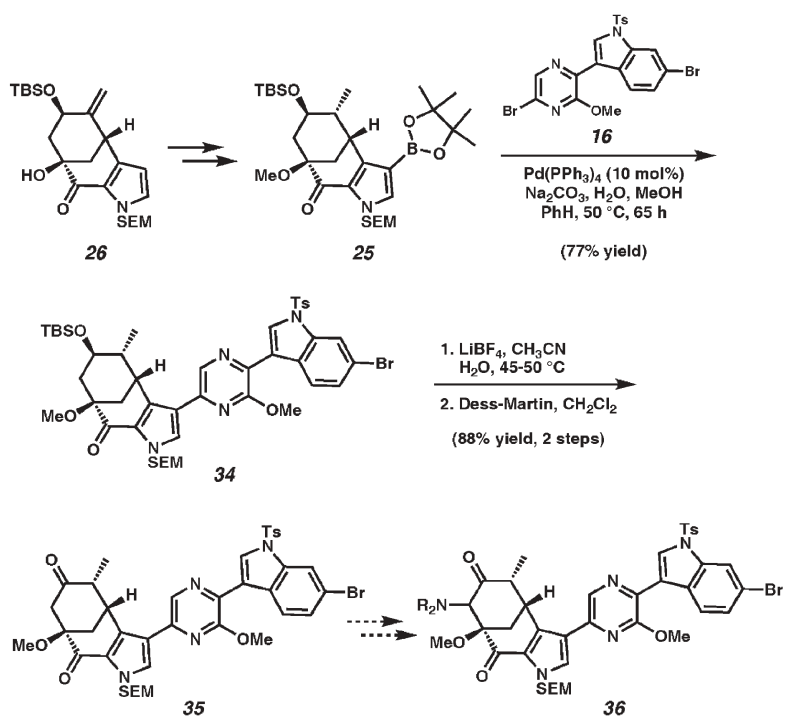

Scheme 8 Synthesis of the carbon scaffold of dragmacidin F.

We anticipated that the presence of an amino group $\alpha$ to the ketone would allow for eventual introduction of the aminoimidazole moiety, as was the case in our synthesis of dragmacidin D (5). Thus, we focused our efforts on preparing compounds related to $\mathbf{3 6}$.

Our first efforts to functionalize the ketone $\alpha$-position involved a nitration strategy to access a compound analogous to an intermediate employed in the dragmacidin D synthesis. ${ }^{37}$ Unfortunately, in all of these cases, formation of the desired nitroketone product was not observed. We switched to a strategy whereby the $\alpha$ nitrogen substituent could be installed via nucleophilic displacement of an $\alpha$ bromide. Thus, bromoketone $\mathbf{3 7}$ was prepared from ketone $\mathbf{3 5}$ as a single diastereomer via a silyl enol ether intermediate (Scheme 9). ${ }^{38}$ Much to our surprise, bromoketone $\mathbf{3 7}$ was extremely prone to base promoted rearrangements when treated with nitrogenous nucleophiles. For example, upon exposure of bromoketone 37 to $\mathrm{NaN}_{3}$, azidoketone $\mathbf{3 8}$ formed as the major product. Furthermore, simply reacting bromide $\mathbf{3 7}$ with a basic fluoride
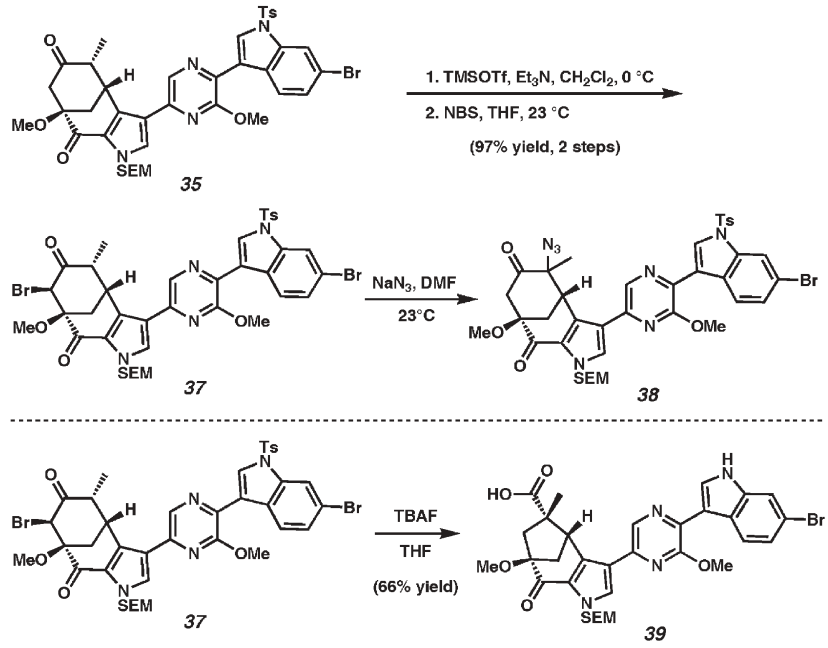

Scheme 9 End-game studies and unexpected rearrangements. 
anion source (TBAF in THF) led to Favorskii rearrangement product 39. ${ }^{39}$ The use of basic amine nucleophiles also yielded related Favorskii products.

A number of other options for installing the $\alpha$-aminoketone, including enol silane oxidative azide formation and aminohydroxylation, were attempted as well, but were met without success. As our options diminished, we considered an alternative plan that would rely on the use of a Neber rearrangement to install the necessary nitrogen substituent. ${ }^{40,41}$ In this scenario, an activated oxime derivative would undergo alkoxide-promoted rearrangement to furnish an $\alpha$-aminoketone. Although this transformation is relatively uncommon and typically hampered by competitive Beckman rearrangement pathways, we elaborated ketone 35 to tosyl oxime 40 via known protocols (Scheme 10). In our initial experiments, exposure of substrate $\mathbf{4 0}$ to aqueous $\mathrm{KOH}$ in ethanol led to the formation of Neber rearrangement products. After optimization, we uncovered a simple two-pot protocol to access deprotected $\alpha$-aminoketone $\mathbf{4 4}$ as a single regio- and stereochemical isomer in excellent yield, as follows: (i) reaction of tosyloxime $\mathbf{4 0}$ with $\mathrm{KOH}$ in ethanol facilitates cleavage of the indole tosyl group and likely promotes formation of azirine 41 which, in turn, is attacked by ethoxide ${ }^{42}$ to afford ethoxyaziridine 42; (ii) subsequent quenching with $\mathrm{HCl}$ results in hydrolysis of the ethoxyaziridine with concomitant partial cleavage of the SEM protective group to furnish aminoketone 43 ; ${ }^{43}$ (iii) treatment of hemiaminal 43 with $\mathrm{K}_{2} \mathrm{CO}_{3}$ removes the remaining portion of the SEM group, thus giving rise to the deprotected product (44). ${ }^{22,44}$ To the best of our knowledge, this is the first example of a successful Neber rearrangement in the context of natural product synthesis. ${ }^{45,46}$

Having installed the $\alpha$-aminoketone and removed the N-protective groups, completion of the total synthesis of dragmacidin $\mathrm{F}$ proceeded smoothly. In order to unveil the masked pyrazinone functionality, Neber rearrangement product 44 was treated with TMSI at $60{ }^{\circ} \mathrm{C}$ (Scheme 11). ${ }^{13}$
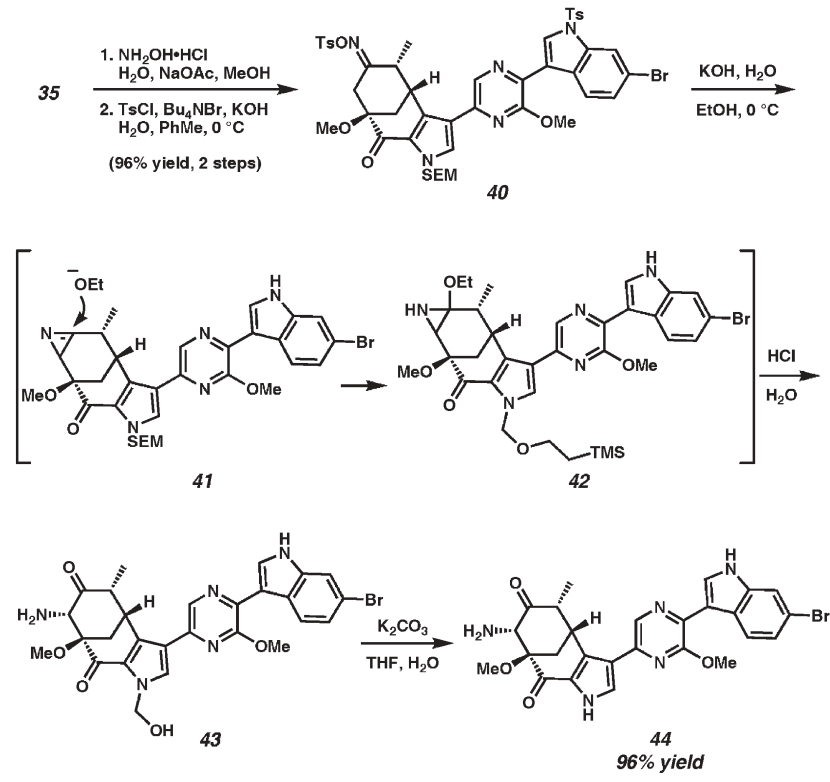

Scheme 10 Neber rearrangement/deprotection sequence.

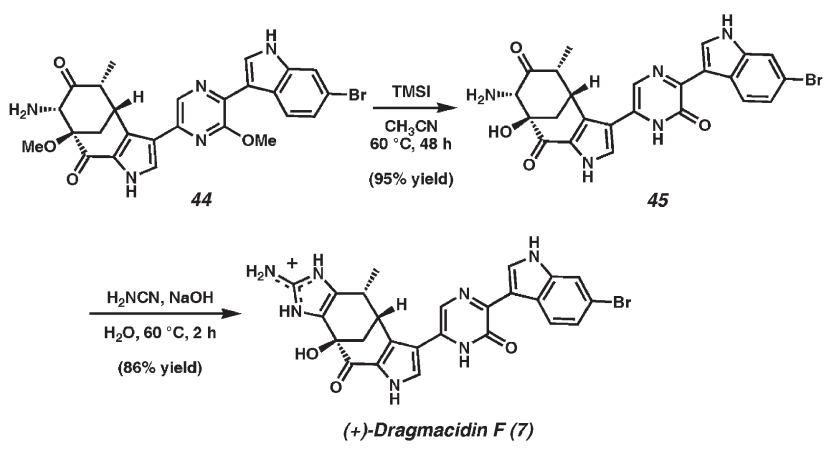

Scheme 11 The total synthesis of (+)-dragmacidin F (7).

Fortuitously, both the pyrazinone and the tertiary alcohol functionalities were revealed simultaneously $(\mathbf{4 4} \rightarrow \mathbf{4 5})$. In the final step of the synthesis, the penultimate aminoketone (45) was subjected to cyanamide and aqueous $\mathrm{NaOH}$ to produce enantiopure dragmacidin F (7). ${ }^{47}$ Our efficient and enantiospecific route allows access to 7 in $7.8 \%$ overall yield in just 21 steps from (-)-quinic acid (28). ${ }^{48}$

As mentioned earlier, the absolute stereochemistry of natural dragmacidin $\mathrm{F}$ was not known when we began our total synthesis. Although our synthetic sample was identical to natural dragmacidin $\mathrm{F}^{3 f}$ by most analytical techniques $\left({ }^{1} \mathrm{H}\right.$ NMR, ${ }^{13} \mathrm{C} \mathrm{NMR}$, IR, UV, HPLC), the sign of optical rotation differed. Thus, we had prepared the unnatural $(+)$ enantiomer of dragmacidin F. However, our synthesis beginning from (-)-quinic acid (28) established, for the first time, the absolute configuration of natural dragmacidin F (7) to be $\left(4^{\prime \prime} S, 66^{\prime \prime} S, 6^{\prime \prime \prime} S\right)$ as shown in Fig. $4{ }^{49}$ In addition, it has been proposed that dragmacidins D, E and F are biosynthetically related. Based on this notion, it is likely that the absolute stereochemical configurations of natural dragmacidins D (5) and $\mathrm{E}(6)$ are $\left(6^{\prime \prime \prime} S\right)$ and $\left(5^{\prime \prime \prime} R, 6^{\prime \prime \prime} S\right)$, respectively.

\section{The total synthesis of nat-(-)-dragmacidin $\mathbf{F}$}

We next pursued the total synthesis of the natural (-) enantiomer of dragmacidin F (7). Having developed a route to (+)-dragmacidin F (7) from (-)-quinic acid (28), we reasoned that the simplest solution to accessing the natural
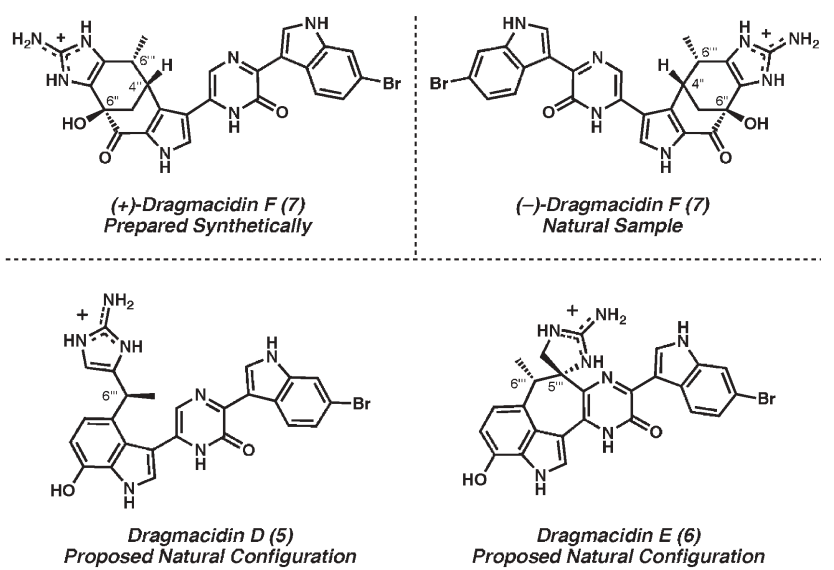

Fig. 4 The absolute stereochemistry of pyrazinone dragmacidins. 
enantiomer of dragmacidin F (7) would be to employ (+)quinic acid (28) as a starting material. Unfortunately, the (+)enantiomer of $\mathbf{2 8}$ is not easily accessible either commercially or via synthetic means. ${ }^{50}$ It seemed plausible, however, that the readily available (-) enantiomer of quinic acid (28) could be used to access both enantiomers of dragmacidin $F(7) .^{51}$ Despite its widespread use in natural product synthesis, (-)quinic acid (28) has rarely been used in an enantiodivergent sense.

Our enantiodivergent strategy to prepare both enantiomers of dragmacidin F from a single enantiomer of quinic acid is shown in Scheme 12. In order to complete the total synthesis of (+)-dragmacidin F (7), a (-)-quinic acid derivative (e.g., 47) had been subjected to a reductive isomerization reaction that proceeded with loss of the $\mathrm{C}(5)$ leaving group. The product of this reaction was elaborated to acyl pyrrole 27 which, in turn, was used to prepare (+)-7. We reasoned that a similar reduction of 47 that occurred with removal of the $\mathrm{C}(3)$ substituent could be used to access cyclohexene 46 en route to (-)-nat-dragmacidin F (7). Since acyl pyrrole species 46 and 27 are diastereomeric, it was also possible that transformations that were used to convert 27 to (+)-7 would not be applicable to the conversion of $\mathbf{4 6}$ to $(-)-\mathbf{7}$. Nonetheless, we focused our attention on developing a reductive isomerization substrate of type $\mathbf{4 7}$ that would lead to cyclohexene $\mathbf{4 6}$.

In order to select the appropriate reductive isomerization substrate needed to access (-)-7, a close examination of the analogous reaction used in our (+)-dragmacidin F (7) synthesis was necessary. It seemed that the success of the reductive isomerization of lactone $\mathbf{2 9}$ to cyclohexene $\mathbf{3 0}$ was largely the result of the excellent carboxylate leaving group at $\mathrm{C}(5)$ of $\mathbf{2 9}$ being restricted to an axial orientation, thereby facilitating its elimination (Scheme 13). Therefore, in designing our new substrate, we envisioned that the tertiary alcohol could be used to lock the $\mathrm{C}(3)$ oxygen substituent in an axial disposition in the form of a carbonate leaving group in order to facilitate its elimination (i.e., $48 \rightarrow 49$ ) ${ }^{30}$ Thus, pyrrolocarbonate 50 was chosen as a suitable (-)-quinic acid derivative needed for our reductive isomerization studies and became our target of interest.

Developing a synthesis of carbonate $\mathbf{5 0}$ was relatively straightforward. (-)-Quinic acid derivative 29, which was used in our total synthesis of (+)-7, was first treated with

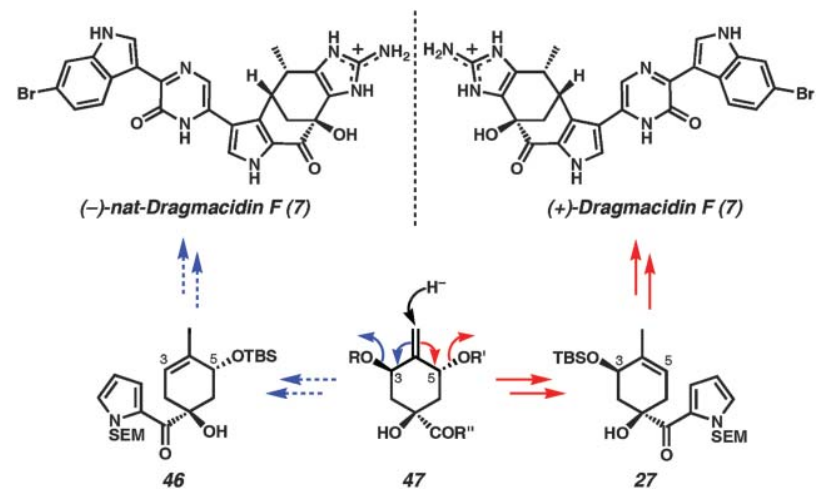

Scheme 12 An enantiodivergent strategy for the preparation of (+)and (-)-dragmacidin F (7).
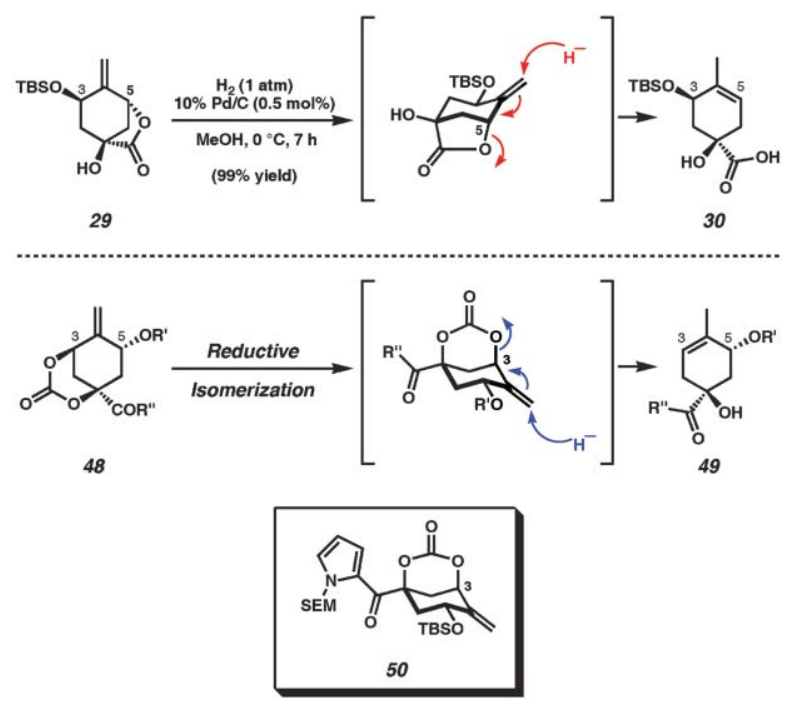

Scheme 13 Rational design of reductive isomerization substrate $\mathbf{5 0 .}$

2-lithio-SEM-pyrrole (32, Scheme 14). Subsequent TBS protection afforded bis(silyl ether) $\mathbf{5 1}$ in good yield. This pseudo- $C_{2}$-symmetric ${ }^{52}$ compound then underwent rapid diastereoselective mono-desilylation upon treatment with TBAF in THF to produce the syn 1,3-diol 52. ${ }^{53}$ Importantly, this desymmetrization proceeded with complete selectivity and allowed us to efficiently differentiate the $\mathrm{C}(3)$ and $\mathrm{C}(5)$ positions of the cyclohexyl moiety. Diol $\mathbf{5 2}$ was smoothly converted to bicyclic carbonate $\mathbf{5 0}$ in the presence of CDI, effectively restricting the $\mathrm{C}(3)$ substituent to an axial disposition. In the key reductive isomerization reaction, exposure of carbonate 50 to our optimal reaction conditions $(2 \mathrm{~mol} \% \mathrm{Pd} /$ $\mathrm{C}, \mathrm{H}_{2}, \mathrm{MeOH}, 0{ }^{\circ} \mathrm{C}$ ) led to the selective formation of the desired cyclization substrate (46) in $90 \%$ yield. $^{54}$

With cyclization substrate $\mathbf{4 6}$ in hand, we focused our attention on the key $\mathrm{Pd}(\mathrm{II})$-mediated carbocyclization to construct the [3.3.1] bicycle of the natural product (Scheme 15). Despite the degree of steric hindrance imposed upon the $C(3) \propto$ face by the bulky $C$ (5) silyl ether group
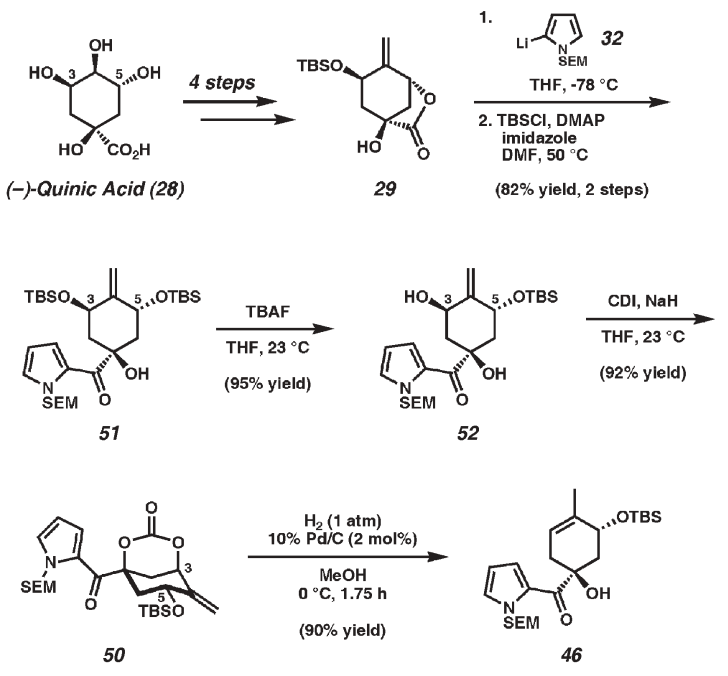

Scheme 14 Elaboration of (-)-quinic acid to 46. 


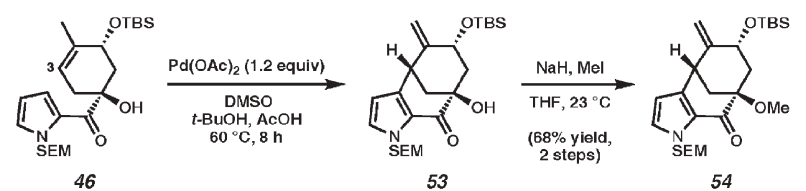

Scheme 15 Construction of [3.3.1] bicycle 54 .

positioned syn to the acyl pyrrole, exposure of $\mathbf{4 6}$ to our optimal cyclization conditions resulted in formation of the desired product (53). Following protection of the tertiary alcohol, [3.3.1] bicycle 54 was obtained in $68 \%$ yield for the two-step process.

After elaboration of bicycle $\mathbf{5 4}$ to boronic ester 55, we were able to assemble the carbon skeleton of (-)-dragmacidin F (56) using our halogen-selective Suzuki coupling methods (Scheme 16). We hoped to convert silyl ether $\mathbf{5 6}$ to ketone $\mathbf{3 5}$, the enantiomer of which was employed in our synthesis of (+)7. Unfortunately, the selective desilylation of $\mathbf{5 6}$ to afford $\mathbf{5 7}$ proved to be difficult. This was surprising, since the diastereomer of $\mathbf{5 6}$ employed in the synthesis of (+)-7 participated in the analogous deprotection reaction.

To overcome this roadblock, we pursued strategies whereby the TBS ether could be cleaved at an earlier stage in the synthesis. In a critical reaction, the sterically less crowded TBS ether of olefinic substrate $\mathbf{5 3}$ underwent smooth and selective cleavage upon treatment with TBAF in THF to afford allylic alcohol 58 (Scheme 17). Upon exposure of alcohol 58 to Brown's cationic rhodium catalyst $\mathrm{Rh}(\mathrm{nbd})(\mathrm{dppb}) \mathrm{BF}_{4}{ }^{55}$ and $\mathrm{H}_{2}$, ketone $\mathbf{5 9}$ formed directly as a single diastereomer in $98 \%$ yield. Regioselective bromination and low-temperature metalation of the pyrrole in the presence of two ketones gave rise to boronic ester 60. Subsequent halogen-selective cross-coupling of $\mathbf{6 0}$ with dibromide $\mathbf{1 6}$ afforded the desired Suzuki adduct (-)-35 (89\% yield). Finally, Suzuki product (-)-35 was converted to (-)-dragmacidin F ((-)-7) via our previously described six-step protocol (vide supra). Synthetic and natural $(-)-7^{3 f}$ were spectroscopically identical, including the sign of optical rotation (natural (-)-7: $[\alpha]_{\mathrm{D}}^{25}-159^{\circ}(c$ 0.4, $\mathrm{MeOH}$ ); synthetic (-)-7: $[\alpha]^{23}{ }_{\mathrm{D}}-148^{\circ}(c \quad 0.2, \mathrm{MeOH})$ ). Our highly efficient syntheses provide (+)-7 in 7.8\% overall yield and (-)7 in $9.3 \%$ overall yield beginning from 28 . Moreover, this work represents the first and, to date, only total synthesis of the antiviral agent dragmacidin $\mathrm{F}^{56}$

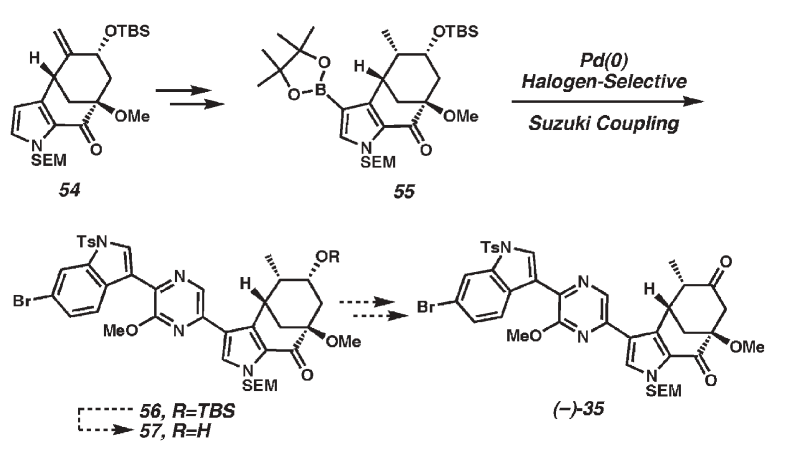

Scheme 16 End-game strategy 1.
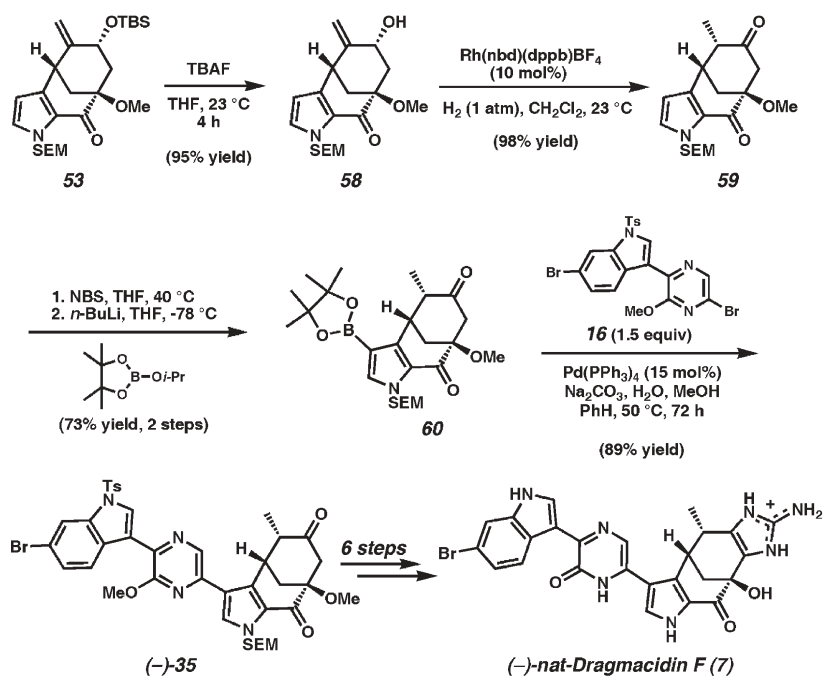

Scheme 17 The total synthesis of (-)-dragmacidin F (7).

\section{The pyrazine dragmacidins: the formal total synthesis of dragmacidins A, B and trans- dragmacidin $\mathrm{C}$}

Having established that halogen-selective Suzuki couplings are a powerful method for constructing the carbon skeleton of the pyrazinone-containing dragmacidins, we hypothesized that a similar strategy could be used to access the piperazine dragmacidins. More specifically, our retrosynthesis for dragmacidins A (2), B (3) ${ }^{3 b}$ and trans-dragmacidin $\left.\mathrm{C} \mathrm{(4)}\right)^{3 c}$ is shown in Scheme 18. Based on the work of Horne and co-workers, ${ }^{4 d, g}$ we envisioned that each of the bis(indole) alkaloids could be accessed from unsaturated pyrazine $\mathbf{6 1}$ in a single step. Pyrazine 61, in turn, would be obtained from two halogenselective Suzuki cross-coupling reactions of boronate $\mathbf{6 2}$ with a dihalogenated pyrazine (63).

In order to probe the limits of our halogen-selective Suzuki cross-coupling methodology, we chose to use known dibromide $64^{57}$ as the critical pyrazine fragment (Scheme 19).

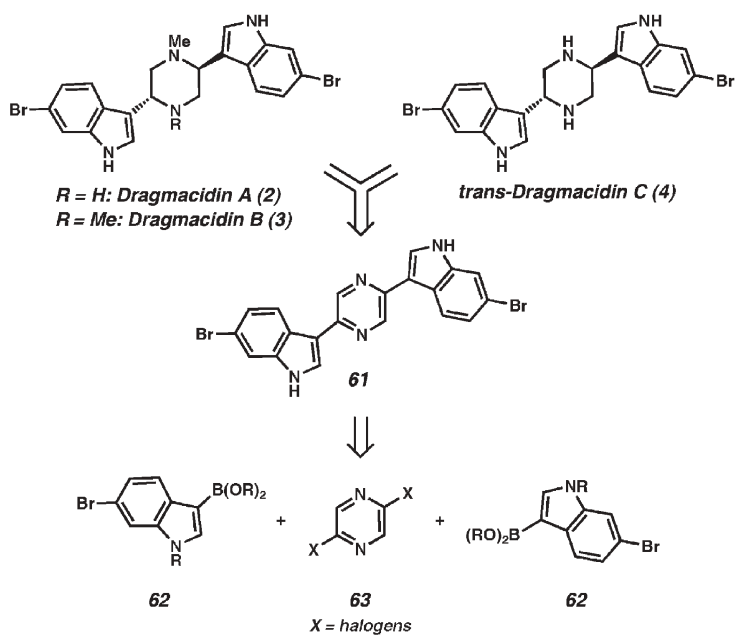

Scheme 18 Retrosynthesis of piperazine dragmacidins. 


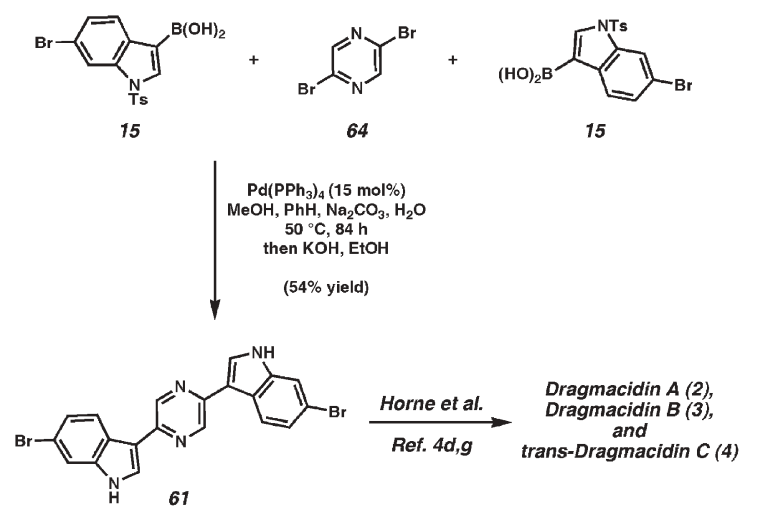

Scheme 19 The formal total synthesis of dragmacidins A (2), B (3) and trans-dragmacidin C (4).

In a one-pot, four-step transformation, an excess of 6-bromoindolylboronic acid (15) was exposed to dibromopyrazine 64 under our standard cross-coupling conditions. Following quenching with $\mathrm{KOH} / \mathrm{ethanol}$, the deprotected pyrazine product (61) was obtained in 54\% yield. Notably, although four bromides were introduced in the reaction mixture, only the two pyrazinyl bromides were reactive in the presence of $\operatorname{Pd}(0)$ at $50{ }^{\circ} \mathrm{C}$. This rapid synthesis of bis(indole)pyrazine $\mathbf{6 1}$ constitutes a formal total synthesis of dragmacidins A (2), B (3) and trans-dragmacidin C (4). ${ }^{4 d, 4 g, 58,59}$ We have also used a similar approach to prepare members of a related family of natural products, the dihydrohamacanthins. ${ }^{60}$

\section{Conclusion}

In summary, we have developed a highly convergent strategy to assemble the dragmacidin natural products. Our approach involves the use of sequential $\mathrm{Pd}(0)$-catalyzed halogen-selective Suzuki couplings to construct the carbon skeletons of these important molecules. Ultimately, this method has been used to achieve the total syntheses of dragmacidins A, B, C and D, in addition to both enantiomers of dragmacidin F. Our work involving dragmacidin D (5) and (+)- and (-)-dragmacidin F (7) remain the only completed total syntheses of the pyrazinone-containing dragmacidins, to date. The success of this work can largely be attributed to the utility of transition metal chemistry in modern organic synthesis, where we have used Pd-mediated cross-couplings, reductive isomerizations and oxidative carbocyclizations as key steps in our total syntheses. Biological evaluation of synthetic dragmacidins and their analogs is currently in progress.

\section{Acknowledgements}

Richmond Sarpong and Daniel Caspi are gratefully acknowledged for their outstanding contributions to the dragmacidin project. The authors thank the NIH-NIGMS (R01 GM6596101), the NDSEG (predoctoral fellowship to N. K. G.), AstraZeneca, Boehringer Ingelheim, Johnson \& Johnson, Pfizer, Merck, Amgen, Research Corporation, Roche and GlaxoSmithKline for generous funding.

\section{Notes and references}

1 (a) J. W. Blunt, B. R. Copp, M. H. G. Munro, P. T. Northcote and M. R. Prinsep, Nat. Prod. Rep., 2004, 21, 1; (b) A. Aygün and U. Pindur, Curr. Med. Chem., 2003, 10, 1113; (c) D. J. Faulkner, Nat. Prod. Rep., 2002, 19, 1; (d) U. Pindur and T. Lemster, Curr. Med. Chem., 2001, 8, 1681.

2 (a) C.-G. Yang, H. Huang and B. Jiang, Curr. Org. Chem., 2004, 8, 1691; (b) Z. Jin, Nat. Prod. Rep., 2003, 20, 584; (c) S. Hibino and T. Choshi, Nat. Prod. Rep., 2002, 19, 148; (d) S. Sasaki, T. Ehara, I. Sakata, Y. Fujino, N. Harada, J. Kimura, H. Nakamura and M. Maeda, Bioorg. Med. Chem. Lett., 2001, 11, 583.

3 For the isolation of the piperazine-containing dragmacidins, see: (a) S. Kohmoto, Y. Kashman, O. J. McConnell, K. L. Rinehart, Jr., A. Wright and F. Koehn, J. Org. Chem., 1988, 53, 3116; (b) S. A. Morris and R. J. Andersen, Tetrahedron, 1990, 46, 715; (c) E. Fahy, B. C. M. Potts, D. J. Faulkner and K. Smith, J. Nat. Prod., 1991, 54, 564. For the isolation of the pyrazinone-containing dragmacidins, see: $(d)$ A. E. Wright, S. A. Pomponi, S. S. Cross and P. McCarthy, J. Org. Chem., 1992, 57, 4772; (e) R. J. Capon, F. Rooney, L. M. Murray, E. Collins, A. T. R. Sim, J. A. P. Rostas, M. S. Butler and A. R. Carroll, J. Nat. Prod., 1998, 61, 660; $(f)$ A. Cutignano, G. Bifulco, I. Bruno, A. Casapullo, L. GomezPaloma and R. Riccio, Tetrahedron, 2000, 56, 3743; $(g)$ A. E. Wright, S. A. Pomponi and R. S. Jacobs, PCT Int. Appl., WO 9942092, August 26, 1999.

4 For synthetic work aimed toward the piperazine-containing dragmacidins, see: (a) B. Jiang, J. M. Smallheer, C. Amaral-Ly and M. A. Wuonola, J. Org. Chem., 1994, 59, 6823; (b) C. R. Whitlock and M. P. Cava, Tetrahedron Lett., 1994, 35, 371; (c) T. Kawasaki, H. Enoki, K. Matsumura, M. Ohyama, M. Inagawa and M. Sakamoto, Org. Lett., 2000, 2, 3027; (d) F. Y. Miyake, K. Yakushijin and D. A. Horne, Org. Lett., 2000, 2, 3185; (e) C.-G. Yang, J. Wang, X.-X. Tang and B. Jiang, Tetrahedron: Asymmetry, 2002, 13, 383; $(f)$ T. Kawasaki, K. Ohno, H. Enoki, Y. Umemoto and M. Sakamoto, Tetrahedron Lett., 2002, 43, 4245; ( $g$ ) F. Tonsiengsom, F. Y. Miyake, K. Yakushijin and D. A. Horne, Synthesis, 2006, 49. For studies targeting dragmacidins D, E or F, see: $(h)$ B. Jiang and X.-H. Gu, Bioorg. Med. Chem., 2000, 8, 363; (i) B. Jiang and X.-H. Gu, Heterocycles, 2000, 53, 1559; (j) C.-G. Yang, J. Wang and B. Jiang, Tetrahedron Lett., 2002, 43, 1063; (k) F. Y. Miyake, K. Yakushijin and D. A. Horne, Org. Lett., 2002, 4, 941; $(l)$ C.-G. Yang, G. Liu and B. Jiang, J. Org. Chem., 2002, 67, 9392; (m) K. S. Feldman and P. Ngernmessri, Org. Lett., 2005, 7, 5449.

5 (a) R. E. Longley, R. A. Isbrucker and A. E. Wright, US Pat., $6,087,363,2000$.

6 (a) M. A. Marletta, J. Med. Chem., 1994, 37, 1899; (b) J. A. Molina, F. J. Jimenez-Jimenez, M. Orti-Pareja and J. A. Navarro, Drugs Aging, 1998, 12, 251; (c) V. Thorns and L. M. Hansen, Exp. Neurol., 1998, 150, 14.

7 W. S. McIntire, D. E. Wemmer, A. Chistoserdov and M. E. Lidstrom, Science, 1991, 252, 817.

8 At the onset of our studies, there was only a single study related to the synthesis of the pyrazinone-containing dragmacidins (5-7). ${ }^{4 h, i}$.

9 Indoles, ed. R. J. Sundberg, Academic Press, San Diego, CA, 1996.

10 For a reference on biomimetic cyclocondensations to produce pyrazinones, see: V. A. Yaylayan, J. Agric. Food Chem., 1996, 44, 2511.

11 For comprehensive reviews on transition metal-catalyzed crosscoupling reactions, see: (a) Metal-Catalyzed Cross-Coupling Reactions, ed. F. Diederich and P. J. Stang, Wiley-VCH, Weinheim, 1998; (b) H. Geissler, in Transition Metals for Organic Synthesis, ed. M. Beller and C. Bolm, Wiley-VCH, Weinheim, 1998, ch. 2.10, p. 158; (c) J. Tsuji, in Transition Metal Reagents and Catalysts, Wiley, Chichester, UK, 2000, ch. 3, p. 27.

12 For an excellent discussion of the cross-coupling chemistry of pyrazines, see: J. J. Li and G. W. Gribble, in Palladium in Heterocyclic Chemistry: A Guide for the Synthetic Chemist, Pergamon, Amsterdam, 2000, pp. 355-373.

13 T. W. Greene and P. G. M. Wuts, Protective Groups in Organic Synthesis, Wiley-Interscience, New York, 3rd edn, 1999.

14 (a) M. P. Edwards, S. V. Ley, S. G. Lister and B. D. Palmer, J. Chem. Soc., Chem. Commun., 1983, 630; (b) E. Piers, R. Britton and R. J. Andersen, J. Org. Chem., 2000, 65, 530 . 
15 Pyrazinyl chlorides coupled competitively with the indolyl bromide functionality.

16 F. Arndt, Org. Synth., 1943, Coll. Vol. 2, 165.

17 (a) G. C. Lancini, E. Lazzari and G. Sartori, J. Antibiot., 1968, 21, 387; (b) P. D. Howes, A. Cleasby, D. N. Evans, H. Feilden, P. W. Smith, S. L. Sollis, N. Taylor and A. J. Wonacott, Eur. J. Med. Chem., 1999, 34, 225.

18 (a) F. A. Luzzio, Tetrahedron, 2001, 57, 915; (b) M. Ayerbe, A. Arrieta and F. Cossio, J. Org. Chem., 1998, 63, 1795.

19 E. J. Gilbert, J. D. Chisholm and D. L. Van Vranken, J. Org. Chem., 1999, 64, 5670.

20 J. Sakowski, M. Bohn, I. Sattler, H. Dahse and M. Schlitzer, J. Med. Chem., 2001, 44, 2886.

21 R. S. Lott, V. S. Chauhan, S. Virander and C. H. Stammer, J. Chem. Soc., Chem. Commun., 1979, 495.

22 Acid-promoted dimerization of the aminoketone functionalities was not observed.

23 N. K. Garg, R. Sarpong and B. M. Stoltz, J. Am. Chem. Soc., 2002, 124, 13179 .

24 Following our publication describing the first total synthesis of dragmacidin D. Jiang reported a similar approach to construct the core of the natural product as summarized below. Ultimately, a mixture of pyrazine products vi-ix was obtained in $61 \%$ combined yield. An account describing the elaboration of vi-ix to the natural product (5) has yet to appear in the literature. See ref. $4 l$.

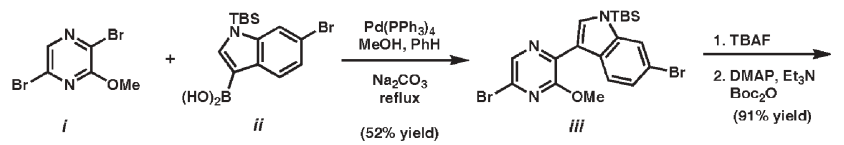

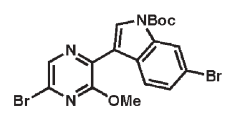

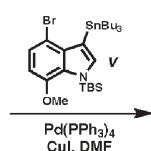
$\mathrm{Cul}, \mathrm{DMF}$
$80^{\circ} \mathrm{C}, 12 \mathrm{~h}$

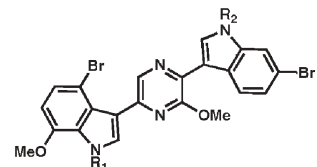

$61 \%\left\{\begin{array}{l}v i, R_{1}=T B S, R_{2}=B o c \\ v i i, R_{1}=T B S, R_{2}=H\end{array}\right.$ yield $\left\{\begin{array}{l}\text { vil, } \mathrm{R}_{1}=\mathrm{TBS}, \mathrm{R}_{2}=\mathrm{H} \\ \text { viii, } \mathrm{R}_{1} \mathrm{H}, \mathrm{R}_{2}=\mathrm{Boc} \\ \text { ix, } \mathrm{R}_{1}=\mathrm{R}_{2}=\mathrm{H}\end{array}\right.$
25 In an attempt to develop an enantioselective route to dragmacidin $\mathrm{D}$, carboxylic acid xii $(90 \%$ ee) was prepared from $\alpha, \beta$-unsaturated acid $\mathbf{x}$ via a Noyori asymmetric hydrogenation reaction. Although carboxylic acid xii could be elaborated to nitroketone 23 without substantial loss in enantiomeric excess $(85 \%$ ee), racemization occurred upon $\mathrm{Sn}(\mathrm{II})$-promoted reduction to aminoketone xiii. It should be noted that the enantiopurity of natural dragmacidin D (5) is somewhat uncertain and remains an open question, as the isolation chemists reported either no optical rotation or small optical rotation values for $\mathbf{5}$; see ref. $3 d$,e .

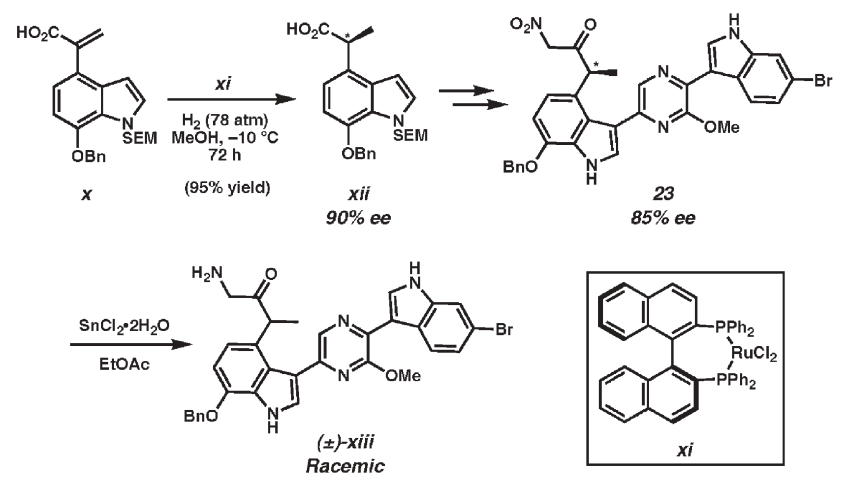

26 For related examples of Pd-mediated carbocyclizations in natural product synthesis, see: (a) P. S. Baran and E. J. Corey, J. Am. Chem. Soc., 2002, 124, 7904; (b) R. M. Williams, J. Cao, H. Tsujishima and R. J. Cox, J. Am. Chem. Soc., 2003, 125, 12172. 27 (a) B. M. Stoltz, Chem. Lett., 2004, 33, 362; (b) R. M. Trend, Y. K. Ramtohul, E. M. Ferreira and B. M. Stoltz, Angew. Chem., Int. Ed., 2003, 42, 2892; (c) E. M. Ferreira and B. M. Stoltz, J. Am.
Chem. Soc., 2003, 125, 9578; (d) H. Zhang, E. M. Ferreira and B. M. Stoltz, Angew. Chem., Int. Ed., 2004, 43, 6144.

28 Following our work, related Pd-mediated cyclizations of pyrroles were reported; see: (a) E. M. Becalli, G. Broggini, M. Martinelli and G. Paladino, Tetrahedron, 2005, 61, 1077; (b) E. M. Beck, N. P. Grimster, R. Hatley and M. J. Gaunt, J. Am. Chem. Soc., 2006, 128, 2528.

29 For reviews and examples regarding the use of (-)-quinic acid in natural product synthesis, see: (a) A. Barco, S. Benetti, C. De Risi, P. Marchetti, G. P. Pollini and V. Zanirato, Tetrahedron: Asymmetry, 1997, 8, 3515; (b) P.-Q. Huang, Youji Huaxue, 1999, 19, 364; (c) S. Hanessian, J. Pan, A. Carnell, H. Bouchard and L. Lesage, J. Org. Chem., 1997, 62, 465; (d) S. Hanessian, In Total Synthesis of Natural Products: The "Chiron" Approach, ed. E. J. Baldwin, Pergamon Press, Oxford, 1983, pp. 206-208.

30 For a discussion regarding this novel reductive isomerization reaction, see: D. D. Caspi, N. K. Garg and B. M. Stoltz, Org. Lett., $2005,7,2513$

31 For the discovery and use of SEM pyrrole, see: (a) J. M. Muchowski and D. R. Solas, J. Org. Chem., 1984, 49, 203; (b) M. P. Edwards, S. V. Ley, S. G. Lister, B. D. Palmer and D. J. Williams, J. Org. Chem., 1984, 49, 3503; (c) M. P. Edwards, A. M. Doherty, S. V. Ley and H. M. Organ, Tetrahedron, 1986, 42, 3723. See also ref. $14 a$.

32 DMSO has commonly been employed in oxidative Pd(II) chemistry. See: (a) R. C. Larock and T. R. Hightower, J. Org. Chem., 1993, 58, 5298; (b) R. A. T. M. Van Benthem, H. Hiemstra, J. J. Michels and W. N. Speckamp, J. Chem. Soc., Chem. Commun., 1994, 357; (c) M. Rönn, J.-E. Bäckvall and P. G. Andersson, Tetrahedron Lett., 1995, 36, 7749; (d) M. S. Chen and M. C. White, J. Am. Chem. Soc., 2004, 126, 1346; (e) S. S. Stahl, Angew. Chem., Int. Ed., 2004, 43, 3400. See also references therein.

33 H. M. Gilow, Y. H. Hong, P. L. Millirons, R. C. Snyder and W. J. Casteel, Jr., J. Heterocycl. Chem., 1986, 23, 1475.

34 Reactions conducted in the presence of acetic acid- $d$ led to deuterium incorporation in the pyrrole ring of both $\mathbf{2 6}$ and $\mathbf{2 7}$, mostly at C(4).

35 For recent reviews of the Heck reaction, see: $(a)$ A. B. Dounay and L. E. Overman, Chem. Rev., 2003, 103, 2945; (b) I. P. Beletskaya and A. V. Cheprakov, Chem. Rev., 2000, 100, 3009; (c) C. Amatore and A. Jutand, J. Organomet. Chem., 1999, 576, 254.

36 (a) Under optimized conditions, intramolecular Heck cyclization of related 3-bromopyrrole derivative xiv resulted in competitive production of undesired product 33. For a detailed account, see: N. K. Garg, D. D. Caspi and B. M. Stoltz, Synlett, 2006, in press; (b) A. F. Littke and G. C. Fu, J. Am. Chem. Soc., 2001, 123, 6989.
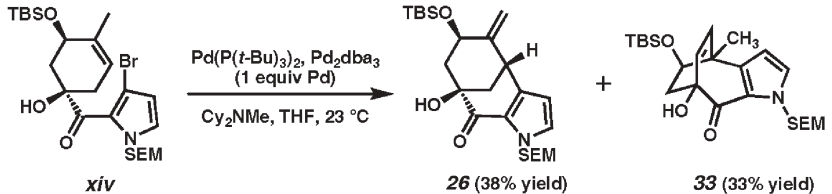

37 (a) R. Rathore and J. K. Kochi, J. Org. Chem., 1996, 61, 627; (b) F. E. Elfehail and W. W. Zajac, Jr., J. Org. Chem., 1981, 46, 5151; (c) F. Elfehail, P. Dampawan and W. Zajac, Synth. Commun., 1980, 10, 929; (d) R. H. Fischer and H. M. Weitz, Synthesis, 1980, 261.

38 W. Kreiser and F. Körner, Helv. Chim. Acta, 1999, 82, 1610.

39 (a) A. E. Favorskii, J. Russ. Phys. Chem. Soc., 1894, 26, 559; (b) P. J. Chenier, J. Chem. Educ., 1978, 55, 286.

40 P. W. Neber and A. V. Friedolsheim, Justus Liebigs Ann. Chem., 1926, 449, 109

41 For a review, see: (a) C. O'Brien, Chem. Rev., 1964, 64, 81; (b) for a recent study involving the Neber rearrangement, see: T. Ooi, M. Takahashi, K. Doda and K. Maruoka, J. Am. Chem. Soc., 2002, 124, 7640.

42 The intermediacy of azirines in Neber rearrangements is well accepted. These azirines presumably arise from transient nitrenes. See: (a) H. O. House and W. F. Berkowitz, J. Org. Chem., 1963, 28, 307; (b) H. O. House and W. F. Berkowitz, J. Org. Chem., 1963, 28, 2271.

43 Hemiaminal $\mathbf{4 3}$ has been observed ${ }^{1} \mathrm{H}$ NMR and mass spectrometry. 
44 Derivatives of $\mathbf{4 0}$ bearing a free tertiary alcohol or a TMSprotected tertiary alcohol produced complex mixtures of products when subjected to Neber rearrangement conditions.

45 (a) Woodward reported a Neber rearrangement during synthetic studies involving lysergic acid. Unfortunately, the Neber rearrangement product could not be further utilized in the synthesis. See: E. C. Kornfeld, E. J. Fornefeld, G. B. Kline, M. J. Mann, D. E. Morrison, R. G. Jones and R. B. Woodward, J. Am. Chem. Soc., 1956, 78, 3087; (b) for the use of the Neber rearrangement in the synthesis of a pharmaceutical substance, see: J. Y. L. Chung, G.-J. Ho, M. Chartrain, C. Roberge, D. Zhao, J. Leazer, R. Farr, M. Robbins, K. Emerson, D. J. Mathre, J. M. McNamara, D. L. Hughes, E. J. J. Grabowski and P. J. Reider, Tetrahedron Lett., 1999, 40, 6739.

46 Related vinyl azide rearrangements have been utilized in the preparation of azirines en route to pyrazines used in the cephalostatin area, see: (a) E. Haak and E. Winterfeldt, Synlett, 2004, 1414; (b) M. Drögemüller, T. Flessner, R. Jautelat, U. Scholz and E. Winterfeldt, Eur. J. Org. Chem., 1998, 2811; (c) M. Drogemuller, R. Jautelat and E. Winterfeldt, Angew. Chem., Int. Ed. Engl., 1996, 35, 1572. We thank Professor Philip L. Fuchs for pointing out these important references.

47 J. C. Boehm, J. G. Gleason, I. Pendrak, H. M. Sarau, D. B. Schmidt, J. J. Foley and W. D. Kingsbury, J. Med. Chem., 1993, 36, 3333

48 N. K. Garg, D. D. Caspi and B. M. Stoltz, J. Am. Chem. Soc., 2004, 167, 9552.

49 Dragmacidin numbering convention, see ref. $3 f$.

50 (a) (+)-Quinic acid $((+)-28)$ is commercially available in limited quantities from Interbioscreen Ltd. (50 mg/\$305 USD); (b) (+)Quinic acid $((+)-\mathbf{2 8})$ potentially could be prepared via multistep synthesis by applying methods used for the preparation of (-)quinic acid (28). See: L. P. Rapado, V. Bulugahapitiya and P. Renaud, Helv. Chim. Acta, 2000, 83, 1625, and references therein
51 (a) G. Ulibarri, W. Nadler, T. Skrydstrup, H. Audrain, A. Chiaroni, C. Riche and D. S. Grierson, J. Org. Chem., 1995, 60, 2753; (b) G. Ulibarri, H. Audrain, W. Nadler, H. Lhermitte and D. S. Grierson, Pure Appl. Chem., 1996, 68, 601; (c) M. T. Barros, C. D. Maycock and M. R. Ventura, J. Chem. Soc., Perkin Trans. 1, 2001, 166.

52 Pseudo- $C_{2}$-symmetric molecules are those that would be $C_{2^{-}}$ symmetric if they did not contain a central chirotopic, nonstereogenic center. For discussions, see: (a) S. L. Schreiber, Chem. Scr., 1987, 27, 563; (b) C. S. Poss and S. L. Schreiber, Acc. Chem. Res., 1994, 27, 9; (c) S. R. Magnuson, Tetrahedron, 1995, 51, 2167; (d) E. Eliel, S. H. Wilen and L. N. Mander, Stereochemistry of Organic Compounds, Wiley-Interscience, New York, 1994.

53 NMR experiments show that the $\mathrm{C}(3)$ silyl ether of $\mathbf{5 1}$ is axially disposed. For similar examples of axial-selective TBS cleavage promoted by TBAF, see: (a) B. N. Craig, M. U. Janssen, B. M. Wickersham, D. M. Rabb, P. S. Chang and D. J. O'Leary, J. Org. Chem., 1996, 61, 9610; (b) R.-M. Meier and C. Tamm, Helv. Chim. Acta, 1991, 74, 807.

54 For a classic example involving the use of conformational analysis to solve stereochemical problems in total synthesis, see: R. B. Woodward, F. E. Bader, H. Bickel, A. J. Frey and R. W. Kierstead, Tetrahedron, 1958, 2, 1

55 (a) J. M. Brown, Angew. Chem., Int. Ed. Engl., 1987, 26, 190; (b) S. H. Bergens and B. Bosnich, J. Am. Chem. Soc., 1991, 113, 958.

56 N. K. Garg, D. D. Caspi and B. M. Stoltz, J. Am. Chem. Soc., $2005,127,5970$

57 H. Ellingson, J. Am. Chem. Soc., 1949, 71, 2798.

58 Treatment of 61 with $\mathrm{NaBH}_{3} \mathrm{CN}$ in formic acid leads to the formation of dragmacidins A (2) and B (3), while the analogous reaction conducted in acetic acid results in production of transdragmacidin C (4).

59 Sakamoto and co-workers have shown that natural dragmacidin C is $c i s$-fused. See ref. $4 f$.

60 N. K. Garg and B. M. Stoltz, Tetrahedron Lett., 2005, 46, 2423. 